\title{
Predictive Analytics im Human Capital Management: Status Quo und Potentiale
}

\author{
Oliver Christ $\cdot$ Nico Ebert
}

Eingegangen: 17. September 2015 / Angenommen: 8. Dezember 2015 / Online publiziert: 23. Dezember 2015

(C) Springer Fachmedien Wiesbaden 2015

Zusammenfassung Unternehmen verfügen mittlerweile über fortgeschrittene analytische Informationssysteme, die es erlauben die wachsenden Datenmengen nahezu automatisiert auszuwerten und Aussagen über zukünftige Entwicklungen zu treffen. Predictive Analytics befinden sie sich im Human Capital Management noch in den Anfängen. Datengetriebene Unternehmen wie Google oder Hewlett-Packard nutzen Predictive Analytics bereits, um Personalbeschaffung und -erhaltung zu verbessern. Obwohl jedoch die Personalbereiche über umfangreiche Daten verfügen, beschränkt sich deren Nutzung nach unserer Erfahrung und einer von uns durchgeführten Befragung in den meisten Fällen immer noch auf reaktives Excel-Reporting und einfachste Prognosen z. B. zur Personalanzahl. Data Mining-Verfahren werden hingegen selten genutzt, obwohl sich daraus für das Human Capital Management und andere Unternehmensbereiche Vorteile ergeben könnten. In diesem Beitrag stellen wir anhand von Praxisbeispielen und ausgewählter Fachliteratur Potentiale von Predictive Analytics im Human Capital Management vor, untersuchen die Verbreitung sowie die Einsatzmöglichkeiten von personalbezogenen Analysen und gehen auch auf die spezifischen Herausforderungen der Nutzung von Personaldaten ein.

Schlüsselwörter Business Intelligence $\cdot$ Human Capital Management · Predictive Analytics · Data Mining

O. Christ ( $\square)$

School of Management and Law, Zurich University of Applied Sciences,

Stadthausstrasse 14,

8401 Winterthur, Schweiz

E-Mail: chrt@zhaw.ch

N. Ebert

LearningCulture $\mathrm{GmbH}$,

Schubertstrasse 9,

8037 Zürich, Schweiz

E-Mail: nico.ebert@gmail.com 
Abstract Nowadays companies have advanced analytical information systems that allow to process the growing amount of data nearly automatically and to predict future developments. While "Analytics" are widespread for example in marketing it is still in its infancy in the domain of Human Capital Management. However, datadriven companies like Google or Hewlett-Packard use Predictive Analytics to optimize staff recruiting and engagement. Despite of the fact that most HR departments have a comprehensive collection of data its use is commonly limited to reactive Excel reports and simple forecasts related to headcount. Advanced techniques such as data mining are seldom used although benefits may derive for the HR department and other departments. In this article we give examples for selected potentials of Predictive Analytics in Human Capital Management and debate the specific challenges of using human resource data.

\section{Einleitung}

Unternehmen sind durch die mittlerweile sehr fortgeschrittenen analytischen Informationssysteme in der Lage, die stetig wachsenden Datenmengen effizient und effektiv zu verarbeiten und semiautomatisch zu analysieren. Eine wichtige Analyseform, die basierend auf vergangenen Daten Aussagen über zukünftige Entwicklungen machen kann, sind Predictive Analytics. Personalabteilungen stehen viele externe und interne Mitarbeiterdaten zur Verfügung und Auswertungen darüber können die Entscheidungsunterstützung für Personalaktivitäten unterstützen und verbessern. Informationstechnisch sehr fortgeschrittene Unternehmen wie Google oder Hewlett-Packard nutzen diese Daten für vielfältige Zwecke, u. a. zur Optimierung von Personalbeschaffung und -erhaltung. In den meisten Unternehmen werden diese Informationen jedoch nur sehr eingeschränkt und mit starken zeitlichen Verzögerungen genutzt. Die Personalabteilungen haben die Wichtigkeit von Predictive Analytics in den letzten Jahren erkannt, dennoch befinden sich analytische Prognose- und Entscheidungsunterstützungsinstrumente in diesem wichtigen Bereich des Unternehmens noch - verglichen mit anderen Unternehmensbereichen wie z. B. Marketing oder der Produktionsplanung und -steuerung - in einer Frühphase (Deloitte 2015, 9 f.).

Für die Personalabteilungen bieten sich neue Möglichkeiten, zukünftige Mitarbeiterperformance, Verhalten, Risiken und Wachstum zu prognostizieren anstatt auf vergangenheitsbasierte Personalkennzahlen zurückzugreifen. Damit das Human Capital Management (HCM) einen strategischen oder auch operativen Mehrwert für das Unternehmen generieren kann, muss es den Abteilungen Informationen an den Ort des Geschehens (z. B. die Produktionshalle oder die Filiale eines Handelsunternehmens) liefern, die sie noch nicht kennen und gleichzeitig Lösungsvorschläge unterbreiten, wie die Geschäftsszenarien gelöst und Potentiale ausgenutzt werden können. Es findet somit eine Veränderung statt, beispielsweise vom reinen Messen der Personalanzahl zur Voraussage, welche Personen das Unternehmen weshalb verlassen werden und wie sich die Abgänge auf das Skill-Portfolio auswirken werden. Die Personalabteilung muss zur Vergrößerung ihres strategischen Wertbeitrages auch Fragen nach der eigenen Effektivität beantworten können. Um diesen Anforderun- 
gen gerecht zu werden und zukünftige Szenarien zu prognostizieren, können Predictive Analytics-Lösungen eingesetzt werden. Nach Auffassung der Beratung Deloitte sind Unternehmen, die Predictive Analytics im den Bereichen Personalbeschaffung, -erhaltung und -entwicklung einsetzen, im Schnitt erfolgreicher als ihre Wettbewerber (Deloitte 2015, $71 \mathrm{ff}$.).

Im folgenden Kap. 2 wird das dieser Arbeit zugrunde liegende Verständnis von Predictive Analytics und HCM erläutert. In Kap. 3 wird der Status Quo von Predictive Analytics im HCM aufgezeigt. Die Darstellung von beispielhaften Szenarien für die Anwendung von Predictive Analytics im HCM findet in Kap. 4 statt. Schließlich fasst Kap. 5 die bisherigen Ergebnisse zusammen und gibt einen Ausblick.

\section{Begriffe}

\subsection{Predictive Analytics}

Analytics wird in diesem Artikel als ein Teilgebiet des „Business Intelligence“ verstanden, d. h. Technologien und Prozesse, die Daten gezielt verarbeiten, um die Leistung und die Entwicklung des Geschäfts zu verstehen, zu prognostizieren und zu bewerten.

Wie in Abb. 1 dargestellt, können wiederum drei Arten von Analytics unterschieden werden (Delen und Demirkan 2013), die unterschiedliche Fragestellungen beantworten:

- „Descriptive Analytics“ nutzt Daten, um die Fragen zu beantworten „Was passiert(e)?“. Dazu werden ad hoc oder periodische Reports aufbereitet, die auch dynamisch und interaktiv sein können (z. B. OLAP, slice/dice). Das Ergebnis von Descriptive Analytics sind Problembereiche und Geschäftsopportunitäten.

- „Predictive Analytics“ versuchen mittels Daten und mathematischen Verfahren erklärende Muster (z. B. Trends, Zusammenhänge, Präferenzen, Cluster) zu erkennen, die zur Prognose genutzt werden können. Die zentrale Frage ist: „Was wird passieren und weshalb?“. Typischen Verfahren sind Data Mining, Text Mining, Web Mining und Prognoserechnungen (z. B. Zeitreihenanalyse). Das Ergebnis der Predictive Analytics sind Prognosen und deren Erklärungen.

Abb. 1 Taxonomie von Analytics (Delen und Demirkan 2013)

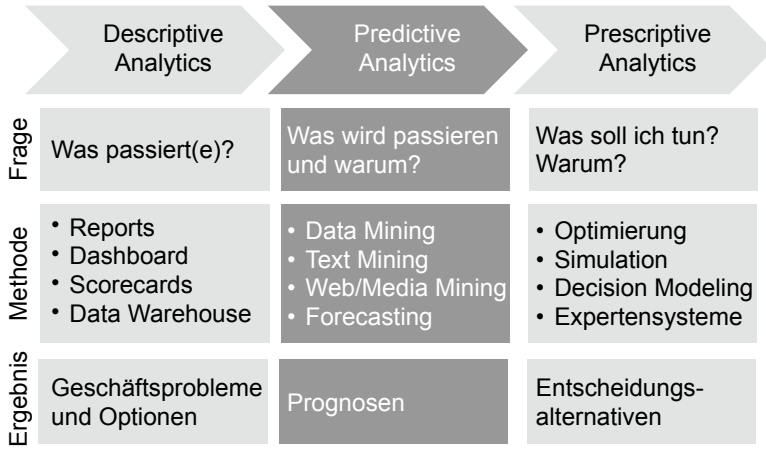


- „Prescriptive Analytics“ nutzen mathematische Methoden, um Handlungsalternativen zu bestimmen. Hierzu werden analog der Theorie rationaler Entscheidungen und Handlungen Ziele, Anforderungen und Rahmenbedingungen vorgegeben und Daten und/oder Expertenwissen genutzt. Typische Methoden sind Lineare Optimierung, Simulation und Experten Systeme. Die Ergebnisse von Prescriptive Analytics sind entweder eine empfohlene Handlungsalternative oder umfangreiche Informationen, die den Handelnden unterstützen.

Eric Siegel (2013, S. 11) definiert Predictive Analytics wie folgt: „Technology that learns from experience (data) to predict the future behavior of individuals in order to drive better decisions." Predictive Analytics sind Technologien, die aufgrund von Vergangenheitsdaten versuchen, zukünftiges Verhalten von Individuen vorauszusagen, damit bessere Unternehmensentscheidungen getroffen werden können. Dabei setzt es das bereits länger existierende Verfahren Data Mining ein, welches automatisierte Techniken für die Analyse von großen Datenbeständen umfasst, um darin Muster und Bedeutungen zu erkennen. Einzuordnen ist es im Segment Business Intelligence und bezweckt dementsprechend die Schaffung von wertvollen Erkenntnissen für die Firma durch Analyse von Rohdaten (PAC 2014). Ursprünglich wurden Predictive Analytics vor allem für die Untersuchung von Kundendaten eingesetzt, jedoch gibt es in der heutigen digitalen Welt immer mehr Anwendungsbereiche, so auch im Personalwesen.

Datenbasierte Prognosen, das Ergebnis von Predictive Analytics, werden in der Wirtschaft seit langem genutzt. Allerdings ermöglicht die steigende Menge verfügbarer und elektronisch zugreifbarer Daten („Big Data“), dass Analysen auch auf solche Bereiche ausgedehnt werden, die sich traditionell auf menschliche Beurteilung und Expertise stützen. Die sinkenden Preise von Rechenleistung und benutzerfreundliche Analyseprodukte sind weitere „Enabler“ für Predictive Analyse (Halper 2014). Typischen Anwendungsgebiete sind z. B. das Marketing (Absatzprognosen, Käuferverhalten), Produktion (Produktions- und Instandhaltungsplanung) oder der Finanzbereich (z. B. Kreditvergabe, Betrugsprognose).

Neben den traditionellen mathematischen und statistischen Methoden (z. B. Regression) werden heuristische Methoden aus dem maschinellen Lernen wie Entscheidungsbäume oder Clustering-Algorithmen zu Erstellung von Modellen zur Prognose genutzt. In jüngster Zeit entwickelte sich zudem die Technik des „Process Mining“ mit dem Ziel, Event-Daten von Geschäftsprozessen zu analysieren, Schwachstellen in den Prozessen frühzeitig zu identifizieren und Prognosen über zukünftiges Prozessverhalten und die Auswirkungen auf den Geschäftsverlauf zu geben. Die Operative Steuerung entlang der Geschäftsprozesse und die Analyse aufgrund verdichteter, analytischer Informationen (Kennzahlen, Prognosen, Frühwarnindikatoren) wachsen aufgrund hochperformanter Systeme und Technologien immer stärker zusammen und ermöglichen die Steuerung des Unternehmens ohne nennenswerte Verzögerungen.

Die Effektivität von Predictive aber auch von Descriptive und Prescriptive Analytics hängt insbesondere von der Qualität der vorhandenen Daten (Genauigkeit, Integrität, zeitnahe Verfügbarkeit), den eingesetzten Werkzeugen sowie den fachlichen, analytischen Fähigkeiten der Anwender ab (Delen und Demirkan 2013). 


\subsection{Human Capital Management}

Der Begriff Humankapital umfasst nach (Meyer-Ferreira 2015, S. 11) das „Wissen, die Fähigkeiten und Erfahrungen von Menschen“ (Meyer-Ferreira 2015, S. 11), es ,gehört den Mitarbeitenden und wird von diesen bis zu einem bestimmten Grad ins Unternehmen investiert“ (Meyer-Ferreira 2015, S. 11). Aufgabe des Unternehmens ist, die effiziente und effektive (im Sinne der Wertschöpfung) Investition des Humankapitals. Aus dieser Aufgabe ergeben sich für die Unternehmensführung drei entscheidende Handlungsfelder des Human Capital Management (Meyer-Ferreira 2015, $31 \mathrm{ff}$.):

- Human Capital Marketing: die Gewinnung der richtigen Mitarbeiter, deren enge Bindung ans Unternehmen und die Förderung ihrer Bereitschaft, das Humankapital ,möglichst vollumfänglich zur Verfügung zu stellen“ (Meyer-Ferreira 2015, S. 31).

- Competence Management: Entwicklung und Transformation individueller Fähigkeiten und individuelles Wissen der Mitarbeiter in wettbewerbsfähige Kompetenzen des Unternehmens.

- Performance Management: Transformation der Unternehmenskompetenzen in wettbewerbsrelevante Leistungen für den Absatzmarkt. In diesem Handlungsfeld erfolgt die strategiekonforme Verzahnung von Personalwesen und operativem Geschäft.

\section{Predictive Analytics im Human Capital Management}

Predictive Analytics Anwendungen befinden sich im Personalbereich noch auf einer frühen Entwicklungsstufe. In einer internationalen Studie unter verschiedenen Unternehmen unterschiedlicher Branchen gaben von 126 Teilnehmern $64 \%$ an, dass in ihrem Marketing-Bereich Predictive Analytics genutzt wird. Nur 17\% gaben hingegen an, dass der Personalbereich Methoden der Predictive Analytics nutzt (Halper 2014). Im Jahr 2014 führten wir eine Befragung von Personalleitern und Personalentscheidern in der Schweiz zum Thema Predictive Analytics durch. Befragt wurden Unternehmen unterschiedlicher Größenordnung und Branche. Fast 50\% der Befragten gaben an, dass die bisher durchgeführten Analysen vor allem reaktiv sind und dem vergangenheitsbezogenen Reporting dienen (Abb. 2). Typische Auswertungen sind z. B. Personalanzahl und -aufwand, Krankenstände oder Mitarbeiterstruktur (z. B. Anteil Männer vs. Frauen, Alter). Lediglich 33\% führen proaktive, fortgeschrittene Analysen durch, z. B. zum Benchmarking. Strategische Analysen (z. B. UrsacheWirkung-Zusammenhänge) oder vorausschauende Analyse (z. B. Risikobewertung, Abgleich mit strategischer Unternehmensplanung) bilden eher die Ausnahme. Die bestehenden Analysen sind vor allem dem Bereich Descriptive Analytics zurechenbar. Die Verfahren aus dem Bereich Predictive Analyse beschränken sich auf einfache Forecasting-Methoden (z. B. Prognosen auf Basis von gleitenden Durchschnitten).

Als Ursache für die geringe Nutzung von Predictive Analytics im HCM geben die Befragten vor allem einen unklaren Mehrwert, fehlendes Know-how bei den 
Abb. 2 Verwendete Analysen in den befragten Unternehmen (eigene Darstellung)

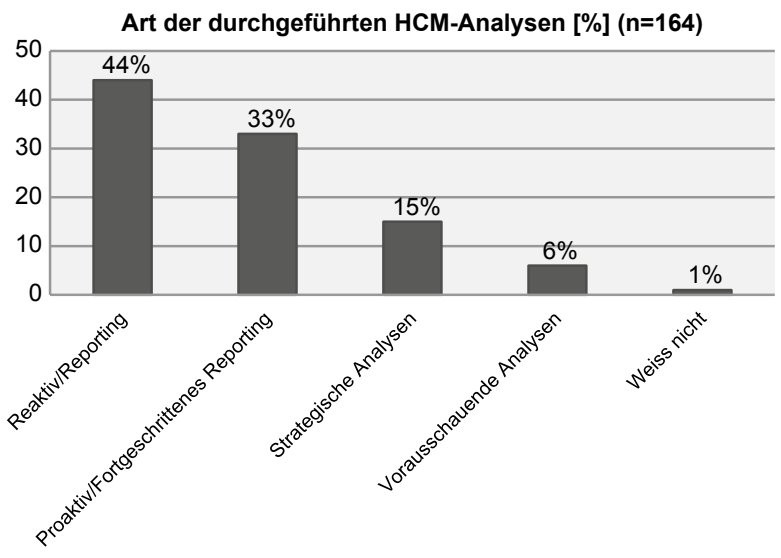

Mitarbeitern sowie eine zu große Komplexität des Themas verbunden mit einer unzureichende Datenbasis an. Potentiale für die Nutzung von Predictive Analytics sehen die befragten Unternehmen vor allen in den Bereichen Personalbeschaffung, Personaleinsatz (insb. Umgang mit Abwesenheiten) und Personalentwicklung (insb. Talentmanagement).

Die zunehmende Menge und Verfügbarkeit von Daten - auch im Personalbereich erlaubt jedoch die Nutzung von komplexeren Predictive Analytics-Methoden jenseits Excel-basierter Forecasts der dargestellten Personalkennzahlen. Im Personalbereich werden in zahlreichen Prozessen Daten produziert. Ob aktive, ausgetretene oder pensionierte Mitarbeitende: alle Daten zu diesen Personen sind im Personalsystem vorhanden. Beispiele sind Abrechnungsergebnisse, Versetzungen, Lohnveränderungen, Mitarbeiterbeurteilungen, Trainingsteilnahmen, Reorganisationen und diverse andere Daten produzierende Aktivitäten. Weiter kommen auch Bewerberdaten und Daten von externen Plattformen dazu. Abhängig von der Häufigkeit der einzelnen Aktivitäten ergibt sich daraus eine große Anzahl Daten, über die Personalverantwortliche verfügen können.

Folgende, beispielhafte Fragen sind für Personalentscheider relevant und können mit Data-, Text- und Web-Mining-Methoden beantwortet werden (Davenport et al. 2010):

- Personalbedarfs- und -strukturplanung: Welchen Einfluss haben Markt- und Industrietrends auf die erforderliche Personalzahl?

- Personalbeschaffung: Welche Bewerber haben die größte Erfolgswahrscheinlichkeit nach der Anstellung? Nach welcher Anstellungszeit stellt sich Erfolg ein? Welche Personen findet man über Soziale Netzwerke, welche besser über andere Kanäle?

- Personaleinsatz: Wie viel Personal wird im Call-Center in Abhängigkeit von Anrufvolumen benötigt? Welche Abteilungen leiden unter schlechter Zufriedenheit der Mitarbeitenden und warum?

- Personalerhaltung: Welchen Faktoren beeinflussen den Austritt eines Mitarbeitenden? Wird ein bestehender Mitarbeitender kündigen? Wie kann dies verhindert werden? Wohin wechseln Mitarbeitende? 
- Personalentwicklung: Welche Trainingsmaßnahmen wirken sich positiv auf die Leistungsfähigkeit der Mitarbeitenden aus? Was sind die Eigenschaften der Leistungsträger? Wie viel Prozent der Mitarbeiter haben in den Jahreszielen die Begriffe „Kostenreduktion“ und „Schulung“?

\section{Praxisfallstudien für den Einsatz von Predictive Analytics im HCM}

\subsection{Google People Analytics (Beispiel Personalbeschaffung und -entwicklung)}

Das datenzentrierte Vorgehen innerhalb der Firma Google umfasst auch deren Personalbereich. Innerhalb der Analytics-Abteilung, die direkt dem Personalchef unterstellt ist, beschäftigen sich über 30 Personen mit unterschiedlichen Fragenstellungen rund um das Thema „People Analytics“ (s. Abb. 3). Dazu zählen Fragen wie „Was macht gute Führungskräfte aus?“ oder ,Wie kann die Diversität innerhalb des Personals erhöht werden?“. Das Leitmotiv des Unternehmens lautet: alle Personalentscheidungen stützen sich auf Daten und Analytics (Davenport et al. 2010; Dekas 2011).

Google bekommt jährlich drei Millionen Bewerbungen, aus denen diejenigen Kandidaten herausgefiltert werden müssen, die über eine hohe berufliche Erfolgswahrscheinlichkeit verfügen und in die Unternehmenskultur passen. Im Jahr 2006 dauerte der Einstellungsprozess noch bis zu sechs Monaten und die Kandidaten mussten zwischen 15-25 Gespräche durchlaufen. Datengestützte Analysen zeigten jedoch, dass vier Gespräche mit den richtigen Personen ausreichend waren, um die spätere berufliche Leistung eines Kandidaten mit einer hohen Konfidenz zu prognostizieren. Dadurch konnte der Einstellungsprozess auf maximal 47 Tage verkürzt werden und die erforderliche Zeit der Angestellten für die Durchführung der Interviews deutlich reduziert werden. Ebenso wurden innerhalb der Firma diejenigen Angestellten identifiziert, deren eingestellte Interviewkandidaten langfristig die beste Leistung zeigten. Diese besonders guten Interviewer wurden wiederum zur Ausbildung anderer Interviewer genutzt (Rafter 2015).

Andere Analysen richten sich auf das Thema Diversität: so wurde festgestellt, das der Frauenanteil in Mittel- und Top-Management-Positionen sehr niedrig war. Während der gesamte Anteil von Frauen bei 30\% lag, waren von den 36 Top-Managern lediglich drei Frauen. Eine Untersuchung kam zu dem Schluss, dass Frauen sich in technischen und Produktmanagement-Jobs viel seltener für Beförderungen vorschlagen lassen. Wenn sie dies jedoch tun, werden sie mit einer höheren Wahrscheinlichkeit befördert als die Männer. In der Folge wurden die Angestellten per E-Mail über die Statistik informiert, was unmittelbar zu einem höheren Anteil von Frauen führte, die sich für die eigene Beförderung einsetzen (Rafter 2015).

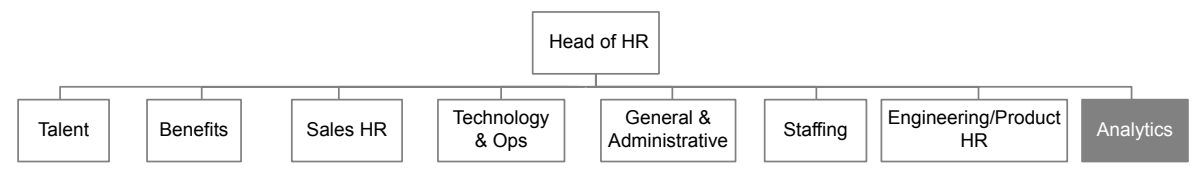

Abb. 3 Organigramm der Google-Personalabteilung (Dekas 2011) 


\subsection{Hewlett-Packard (Beispiel Personalerhaltung)}

Das US-amerikanische Technologienunternehmen wollte das Problem der Personalfluktuation adressieren. In einigen Bereichen der Firma verließen laufend Angestellte das Unternehmen, was zu hohen Rekrutierungskosten führte. Das Ziel war die Entwicklung einer „Flight Risk Score“, d. h. eines Wertes je Mitarbeitenden, der die Wahrscheinlichkeit widerspiegelt, das Unternehmen zu verlassen (Siegel 2013, S. 37). Zu diesem Zweck wurde ein Pilotprojekt in einer Abteilung mit ca. 300 Mitarbeitern durchgeführt, die Vergütungen von Vertriebspersonal bearbeiten. Die jährliche Fluktuation in dieser Abteilung belief sich auf rund $20 \%$ und aufgrund der sehr spezialisierten Aufgabe, waren die Kosten für die Ersetzung ehemaliger Angestellter sehr hoch. Aus der Analyse der Daten der Angestellten wurde ersichtlich, dass Angestellte mit höheren Gehältern, kontinuierlicheren Gehaltserhöhungen und guten Leistungsbewertungen über eine geringere „Flight Risk Score“ verfügen. Ebenso wirkte sich bei einseitigen Arbeiten die Job-Rotation positive auf die Rate aus. Erstaunlicherweise wirkten sich Beförderungen jedoch nicht zwangsläufig positiv auf die Rate aus: waren die Promotionen nicht mit Gehaltserhöhungen verknüpft, führten häufige Beförderungen zu einer hohen Kündigungswahrscheinlichkeit. Nach der Pilotphase wurde die Flight Risk Score auf alle 350.000 Angestellten des Konzerns ausgedehnt (Siegel 2013, 49 ff.).

\subsection{Starbucks (Beispiel Personalbeschaffung und -erhaltung)}

Ebenso wie Hewlett-Packard war der amerikanische Kaffee-Einzelhändler mit 190.000 Angestellten ebenfalls mit einer hohen Personalfluktuation konfrontiert. Da in der Branche vielfach Studierende angestellt werden, die selten lange bei einem Unternehmen arbeiten, liegt der Branchendurchschnitt für die Fluktuation bei $140 \%$ pro Jahr. Um die Ansichten und Probleme innerhalb der Belegschaft besser zu verstehen, wurden die Kommentare aktueller und ehemaliger Mitarbeitende auf der Arbeitgeberbewertungsplattform Glassdoor.com von einem externen Unternehmen analysiert (Sakellariadis 2015).

Zwar ist die Bewertung von Starbucks mit 3.8/5 Sternen auf Glassdoor.com grundsätzlich positiv, allerdings können daraus keine unmittelbaren Schlüsse auf die Ansichten der Mitarbeitenden gezogen werden. Daher wurden mit einer Text-Mining-Methode über 5000 Kommentare der Nutzer analysiert und die Aussagen zu verschiedenen stereotypen Clustern, sogenannten Erzählungen, gruppiert und bewertet (Abb. 4). Die Erzählungen stehen stellvertretend für unterschiedliche Haltungen der Angestellten und reichen von „Starbucks ist der Stern“ bis „Glorifiziertes Fastfood“. Selbst in eher negativen Berichten werden Mitarbeiter-Leistungen, wie kostenlose Getränke, gelobt. Gleichzeitig werden jedoch in einigen Beschreibungen die intransparente Beförderungspraxis durch das Mittelmanagement und die schlechten Karrieremöglichkeiten kritisiert (Sakellariadis 2015). 


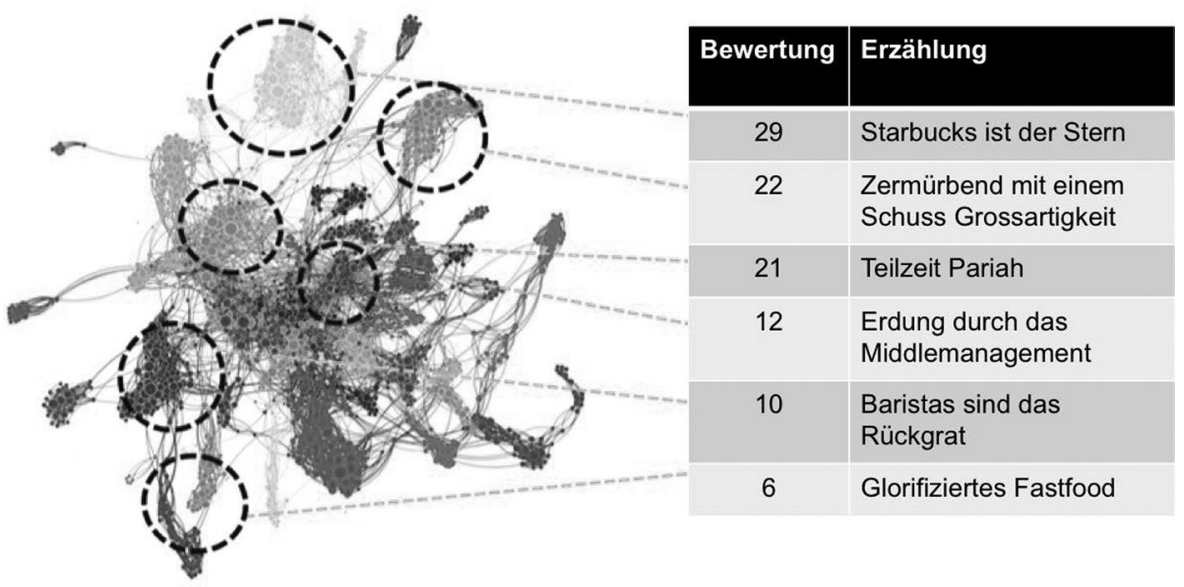

Abb. 4 Clustering der Starbucks-Angestelltenkommentare zu Erzählungen (Sakellariadis 2015)

4.4 Globales Industrieunternehmen (Beispiel: Personalbedarfs- und -einsatzplanung)

Ein global tätiges Industrieunternehmen mit Stammsitz in Deutschland hat sich entschieden, die Personalbedarfs- und -einsatzplanung im produzierenden Bereich zu professionalisieren und mit Data Mining-Unterstützung zielgerichtet entlang des Produktionsprozesses zu organisieren. Dazu werden die Vergangenheitsdaten aus den Zeiterfassungssystemen, den Personalsystemen und den auswertungsrelevanten ERP-Modulen zusammengeführt, harmonisiert und mit externen Daten (insb. Wetterdaten, Ferienkalender) angereichert. Die verdichtete Datenbasis dient als Prognosegrundlage für die Vorhersage des zukünftigen Personalbedarfs in der Produktion. Mittels Predictive Analytics-Instrumenten werden die Abhängigkeiten der Vergangenheit mit den zukünftigen Ferienkalendern, Wetterdaten und Produktionsplänen abgeglichen und ein qualitativer und quantitativer Personalbedarf für einen bestimmten Zeitraum ermittelt und kann so frühzeitig erkannt und bedient werden. So lässt sich bspw. aufgrund der Verbindung von Daten zum Familienstand der Mitarbeiter, den Ferienkalendern und den vergangenen Fehltagen, genau vorhersagen, wie viel Mitarbeiter in welchen Abteilungen in welcher Periode fehlen werden und Engpässe können frühzeitig durch Anpassungen der Produktionsplanung oder der Personaleinsatzplanung vermeiden werden. Ein weitergehendes Szenario wäre die Vorhersage von Abwesenheiten bei bestimmten Wetterlagen oder Großevents wie Fußball-WM. Datenintegration, effiziente Data Mining-Lösungen und die Verbindung der Personal- und der Produktionssicht ermöglichen so eine effektive Personaleinsatzplanung in der Produktion. 


\section{Herausforderungen und Rahmenbedingungen}

\subsection{Strategie und Organisation}

Im Zusammenhang mit der konkreten Umsetzung einer „Analytics-Strategie“ sind für viele Unternehmen der Mangel an qualifizierten Analysten und ein fehlendes Verständnis für die Anwendung von Predictive Analytics die zentralen Herausforderungen (Halper 2014, S. 12). Am Beispiel der Google People Analytics-Abteilungen (vgl. Abschn. 4.1) wird deutlich, dass für die Umsetzung von Predictive Analytics im HCM neben betriebswirtschaftlichen Qualifikationen auch Fähigkeiten in den Bereichen Psychologie, Statistik, Mathematik und Informatik erforderlich sind. Für die Personalabteilungen bedeutet dies, dass das Wissen häufig noch aufgebaut werden muss - sei es in Form von Einstellung neuer oder Weiterbildung bestehender Mitarbeiter (vgl. (Davenport et al. 2010)). Dieses Defizit wurde auch durch unsere Befragung unter Führungskräften aus dem Personalwesen in der Schweiz bestätigt. Mittlerweile wurde in einigen Unternehmen eine dedizierte Analytics-Abteilung innerhalb der Personalabteilung etabliert (Vgl. Fallbeispiel Google), allerdings ist dies vermutlich bisher die Ausnahme.

Neben qualifiziertem Personal ist auch die Unterstützung durch die Führungskräfte des Unternehmens und der Personalabteilung erforderlich. Zu dieser Kultur gehört auch, dass die Personalabteilung je nach Unternehmensstrategie Ziele für die Analyse-Aktivitäten definiert und Personaldaten und -analysen mit anderen Abteilungen teilt (Davenport et al. 2010).

\subsection{Daten und Technik}

Viele größere Unternehmen verfügen über ein Data Warehouse, das Kennzahlen für das Personalcontrolling enthält, dennoch stellt die Datenintegration eine Herausforderung für Unternehmen dar (IBM 2008, S. 40; Halper 2014, S. 19). Zu einem müssen - unter Umständen auch unstrukturierte - Datenquellen erschlossen werden (z. B. Qualifikationsprofile) und zum anderen müssen die in den letzten Jahren populären Software as a Service-Systeme (z. B. für die Personalbeschaffung) integriert werden (Deloitte 2015, S. 75). Aufgrund der zum Teil sensiblen Personaldaten ist im Einzelfall zu entscheiden, welche Daten integriert werden dürfen und wer zugriffsberechtigt. Ebenso ist die Qualität der Daten ein wichtiges Kriterium für den Erfolg von Predictive Analyse, weswegen die Data Governance des Unternehmens auf diese ausgedehnt werden sollte (vgl. (Halper 2014, S. 20)). Schließlich sollten die eingangs dargestellten klassischen Kennzahlen des Personalcontrollings um neue Metriken wie die „Flight Risk Score“ (vgl. Abschn. 4.2) erweitert werden, welche die zusätzlichen Möglichkeiten der Predictive Analytics widerspiegeln (Davenport et al. 2010).

\subsection{Recht}

Bei der Umsetzung von Predictive Analytics-Projekten müssen, ebenso wie bei andere IT-Projekten im HCM-Umfeld, die länderspezifischen datenschutzrechtlichen Grundlagen beachtet werden. In Deutschland sind dies unter anderem das Betriebs- 
verfassungsgesetz und das Bundesdatenschutzgesetz. Entscheidend ist zunächst die Frage, ob die Daten, die gespeichert, verarbeitet oder genutzt werden, personenbezogen sind. Dies ist nicht der Fall, wenn die Daten keinen Rückschluss auf einzelne Personen zulassen (vgl. Abschn. 4.3) und kann auch im Nachhinein durch die Anonymisierung personenbezogener Daten gewährleistet werden. Werden jedoch personenbezogene Daten verarbeitet, gilt das Recht auf informationelle Selbstbestimmung der Betroffenen und ihre Einwilligung zur Verarbeitung ist erforderlich (Gola 2015, 127 ff.).

Das Bundesdatenschutzgesetz erlaubt explizit die Erhebung, Verarbeitung und Nutzung im Rahmen der Einstellung, Weiterbeschäftigung oder Beendigung eines Arbeitsverhältnisses (vgl. Abschn. 4.1). Ebenso zugelassen ist das Scoring der Mitarbeitenden analog zum Fallbeispiel Hewlett-Packard. Grundsätzlich empfiehlt sich in Predictive Analytics-Projekten mit personenbezogenen Daten jedoch immer die frühzeitige rechtliche Abklärung sowie das Involvieren von Betriebsrat und betrieblichem Datenschutzbeauftragen (Gola 2015, 139 ff.).

\section{Fazit und Ausblick}

Der Einsatz von Predictive Analytics und die enge Verzahnung von operativen und analytischen Systemen sind im HCM im Vergleich mit anderen Prozessen und Organisationseinheiten noch in einer frühen Phase. Die dargestellten Fallstudien der "Early Adopter" im HCM sowie der Vergleich mit fortgeschrittenen Bereichen wie der Warenbewirtschaftung, der Sortimentsgestaltung oder der Produktionsplanung können Möglichkeiten der Weiterentwicklung aufzeigen. Auf technologischer Ebene ergeben sich durch den Einsatz innovativer Lösungen und Konzepte wie In-MemoryComputing oder linguistische Textanalyseverfahren ebenfalls interessante Möglichkeiten für die HCM-Abteilungen und Prozesse der Unternehmen.

Zum heutigen Zeitpunkt ist die Organisation des HCM größtenteils prozessorientiert und vielfach durch Standardsoftwarepakete unterstützt. Analytische Informationen, insbesondere Predictive Analytics werden noch nicht ausreichend in die Personal- und die Leistungsprozesse der Unternehmen eingebunden. Ein Grund für die Zurückhaltung könnte in der Sensitivität der Personaldaten und der dadurch resultierenden Komplexität der Datenorganisation liegen. Zukünftige PredictiveAnalytics-Lösungen müssen daher eine Balance zwischen Transparenz und Schutz der persönlichen Daten schaffen und die Datenauswertungen so organisieren, dass diese rechtlichen und ethischen Standards genügen.

Für Unternehmen ergeben sich Anwendungsmöglichkeiten für Predictive Analytics im Human Capital Management in allen drei oben genannten Handlungsfeldern des HCM (Human Capital Marketing, Competence Management und Performance Management). Da diese drei Felder eng miteinander verbunden sind, bietet es sich an, ausgewählte Analysen prozess- und bereichsübergreifend einzusetzen. Aufgrund der Sensitivität der in vielen Anwendungsfällen benötigten personenbezogenen Daten, bietet es sich an, zunächst solche Szenarien auszuwählen, die auch von anonymen und nicht personenbezogenen Daten profitieren. Als Vorgehen empfiehlt sich, mit diesen weniger sensitiven Einsatzbereichen zu beginnen und zu evaluieren, an 
welchen Stellen Bedarf für vorausschauende Analysen besteht und diese Analysen eng mit den Kernprozessen des HR und des Unternehmens zu verzahnen. In diesem Bereich wird in den nächsten Jahren Bedarf an Methoden, Vorgehensmodellen und an Forschungsarbeiten bestehen, die auch von den Autoren und deren Institutionen begleitet werden.

\section{Literatur}

Davenport TH, Harris J, Shapiro J (2010) Competing on talent analytics. Harv Bus Rev 88(52-8):150

Dekas K (2011) People analytics: using data to drive HR strategy and action. In: O'Reilly. https://www. youtube.com/watch?v=16ISTjupi5g. Zugegriffen: 14. Aug. 2015

Delen D, Demirkan H (2013) Data, information and analytics as services. Decis Support Syst 55:359-363. doi:10.1016/j.dss.2012.05.044

Deloitte (2015) Global Human Capital Trends 2015. http://www2.deloitte.com/us/en/pages/human-capital/articles/introduction-human-capital-trends.html. Zugegriffen: 18. Aug. 2015

Gola P (2015) HR Intelligence und Analytics - Datenschutzrechtliche Grenzziehungen. In: Strohmeier S, Piazza F (Hrsg) Human Resource Intelligence und Analytics. Springer Fachmedien Wiesbaden, Wiesbaden, S 127-158

Halper F (2014) Predictive analytics for business advantage

IBM (2008) Global Human Capital Study. IBM Corporation

Meyer-Ferreira (2015) Human Capital strategisch einsetzen - Neue Wege zum Unternehmenserfolg. Luchterhand-Verlag, München

PAC (2014) Predictive Analytics - Mehrwerte, Einsatzbeispiele und Herausforderungen. http://www.sas. com/de_de/whitepapers/ba-wp-pac-predictive-analytics-2308002.html. Zugegriffen: 10. Sept. 2015

Rafter MV (2015) Laszlo Bock: Just Google Him. In: Workforce. http:/www.workforce.com/articles/ 21168-laszlo-bock-just-google-him. Zugegriffen: 14. Aug. 2015

Sakellariadis S (2015) Making Sure the Cup Stays Full at Starbucks: Leveraging Narratives from Glassdoor.com to Improve Recruitment and Retention. In: Huffingtonpost. http://www.huffingtonpost. com/sophie-sakellariadis/making-sure-the-cup-stays_b_7935760.html. Zugegriffen: 14. Aug. 2015

Siegel E (2013) Predictive analytics: the power to predict who will click, buy, lie, or die. Wiley, Hoboken 